\title{
Elevating the Behavioral and Social Sciences in Premedical Training: MCAT2015
}

\author{
Laura Weiss Roberts • Alan K. Louie • Michelle Goldsmith • \\ Glendon R. Tait • Richard Balon • Eugene V. Beresin • \\ Sylvia Bereknyei • John H. Coverdale
}

Received: 13 January 2015 / Accepted: 21 January 2015 / Published online: 21 February 2015

(C) Academic Psychiatry 2015

Beginning in April 2015, students seeking admission to medical school will take the fifth version of the Medical College Admission Test (MCAT). The MCAT2015 will in many respects resemble the MCAT1991, but differences between the two tests are important, especially for the profession of psychiatry. The MCAT2015 is in part a response to "outreach and survey data [that] highlighted ethics, philosophy, cultural studies, and population health as important new disciplines to include" [1, p. 563]. The new exam will continue to emphasize basic biomedical sciences and yet will give greater attention to "concepts that tomorrow's doctors need to know in order to serve an increasingly diverse population and have a clear understanding of the impact of behavior on health" [2]. As the Association of American Medical Colleges (AAMC) [3] states regarding the new exam:

"The natural sciences sections reflect recent changes in science and medical education; the addition of the social and behavioral sciences section recognizes the importance of socio-cultural and behavioral determinants of

L. W. Roberts $\cdot$ A. K. Louie $(\triangle) \cdot$ M. Goldsmith $\cdot$ S. Bereknyei Stanford University, Stanford, CA, USA

e-mail: louiemd@stanford.edu

G. R. Tait

Dalhousie University, Halifax, NS, Canada

R. Balon

Wayne State University, Detroit, MI, USA

E. V. Beresin

Harvard Medical School, Boston, MA, USA

J. H. Coverdale

Baylor College of Medicine, Houston, TX, USA health and health outcomes; and the Critical Analysis and Reasoning Skills section reflects the value that medical schools place on analysis, evaluation, and reasoning skills and on broad preparation for medical school."

The new exam will have four sections, each producing a separate score: (1) Biological and Biochemical Foundations of Living Systems; (2) Chemical and Physical Foundations of Biological Systems; (3) Psychological, Social, and Biological Foundations of Behavior; and (4) Critical Analysis and Reasoning Skills. The writing sample has been eliminated from the MCAT2015, and the Verbal Reasoning section has been replaced with the Critical Analysis and Reasoning Skills section $[4,5]$.

The MCAT2015 will have more questions and take approximately $7.5 \mathrm{~h}$ to administer [6]. The new exam also adds new content beyond the natural sciences that have traditionally dominated the exam. For instance, the Psychological, Social, and Biological Foundations of Behavior section will introduce psychology, sociology, and related sciences such as epidemiology and biostatistics where content is relevant to health. The Critical Analysis and Reasoning Skills section "draws on passages from the social sciences and humanities disciplines, including passages from ethics, philosophy, studies of diverse cultures, and population health" [7]. In addition, the MCAT2015 tests a candidate's ability to examine research methods and fundamentals of interpreting data and to think critically in both the natural and social sciences. For instance, a question regarding data interpretation might ask students to draw conclusions and inferences from visual representations of results, such as graphs of the results of a social psychology experiment. The test has been redesigned to encourage students to apply their knowledge like a practicing scientific physician, requiring them to think about physiology, pathology, 
and psychosocial factors of disease and wellness as applied to patient care, public health, populations, and research [4].

The goals of this editorial are to comment on some of the possible implications of these changes in the MCAT2015 for the practice of psychiatry and medicine in general. Specifically, we will comment on how an elevation of the behavioral and social sciences in premedical training should enhance the culture of medicine as a biopsychosocial enterprise. We will also speculate on how the MCAT2015 might contribute to humanistic behaviors in the practice of medicine and to recruitment to psychiatry.

\section{Enhancing the Biopsychosocial Model of Medical Practice}

\begin{abstract}
"The most common criticism made at present by older practitioners is that young graduates have been taught a great deal about the mechanism of disease, but very little about the practice of medicine-or, to put it more bluntly, they are too 'scientific' and do not know how to take care of patients." Francis W. Peabody [8, p. 877]
\end{abstract}

Peabody's [8] words from 1927 are still astonishingly applicable today. The care of patients requires more than the narrow scientific perspective we have fostered, unwittingly, to the neglect of more humanistic practice. The biopsychosocial approach is not fostered by the current practice of medicine that is based on a reductionist or solely biophysiochemical model. The biopsychosocial model [9], however, has become an imperative as medicine finds itself in an increasingly complex and global society.

The MCAT2015 may prove to be a clever strategy to rebalance the books, as it were, in favor of a biopsychosocial approach to medicine. First, the MCAT2015 will implicitly communicate that the behavioral and social sciences are among the foundational sciences of medicine, alongside the natural sciences. Second, in preparing for the MCAT, premedical students will likely enroll in college courses that touch upon behavioral and social sciences, and thus people entering medical school will be more knowledgeable in these areas. Third, the inclusion of behavioral and social sciences and humanities will influence the MCAT score that has often been used by medical school admissions committees as a threshold metric during the initial screening of applications. These three influences will be expanded on below, in turn.

Acknowledging the importance of the behavioral and social sciences will not only underscore the importance of a biopsychosocial model, but also an ethical approach to the practice of medicine. Physicians of tomorrow must prepare to take account of and manage the environment and psychosocial factors that contribute to ill health and create barriers to treatment. This includes awareness of cultural disadvantages, interplay of social institutions with health, impoverishment and homelessness, mental illness, traumatic experiences, motivations for health behavior and attitudes, and the vulnerabilities of special groups that contribute to discrimination and impede access to health care across the globe.

The MCAT2015 is an exam that demands that a student be knowledgeable in many areas - natural sciences (biology, chemistry, and physics), mathematics (including more biostatistics and research design), and now awareness of healthrelated knowledge in the behavioral and social sciences and the humanities. Colleges have already been designing coursework to integrate content across disciplines to prepare students for the new test format and to help them become better doctors. Faculty members at several medical schools have been developing innovative undergraduate courses for achieving this goal $[3,4]$. Students will want to know what behavioral and social science knowledge they will need to master for the exam, but defining this may be difficult, especially in comparison to the curriculum of organic chemistry, for example, which is more standardized throughout the United States. The AAMC [10] and the Khan Academy [11], through support from the Robert Wood Johnson Foundation, have provided a platform for current medical students to develop relevant video tutorials and training videos on short, succinct topics in preparation for the MCAT2015. These topic-specific videos are freely available and accessible to the public through the Internet, thus equalizing access to study materials across learning environments, socioeconomic levels, and demographics. These and similar preparatory materials include psychology and sociology content in addition to the traditional natural sciences.

Changes in the MCAT2015 may be another step toward the more holistic evaluation of medical school applicants. In 2007 the AAMC established the Holistic Review Project to create and sustain medical applicant diversity [12]. Holistic review is defined as "a flexible, individualized way of assessing an applicant's capabilities by which balanced consideration is given to experiences, attributes, and academic metrics and, when considered in combination, how the individual might contribute value as a medical student and physician" [13]. Greater consideration of applicants who have not followed the usual "pre-med" academic path is a significant part of a holistic admission process [3]. The new behavioral and social science sections of the MCAT2015 may help assess how well rounded an applicant's knowledge is beyond the natural sciences. The exam, symbolic of the medical school application process, may be more attractive to individuals from varied academic and demographic backgrounds [4]. Thus, the hope is that the MCAT2015 may be one factor in changing admission policies to diversify student bodies in medical schools. A testable hypothesis is that improving the status of the behavioral and social sciences within medicine may result in a more socially and ethnically diverse and idea-rich workforce. 
A more diverse study body may be better prepared, interested, and capable in the behavioral and social sciences in medical school. This might have a bottom-up influence on faculty, curriculum, and values in medical education and training. In short, we might see more of the biopsychosocial model incorporated into future identities, roles, and critical thinking skills of physicians. This could clearly have effects that transcend the admission process and change the very ethos of medicine. We might also hope that the increased sensitivity of medical students and future physicians to psychosocial factors will lead to an increase in the visibility of patients' mental health needs, as encountered in primary care and across all specialties of medicine. Psychiatrists should be ready to step up and lead medical schools in teaching not only about mental disorders but also about the psychosocial contributors to all diseases and health.

\section{Enhancing Humanism}

"One of the essential qualities of the clinician is interest
in humanity, for the secret of the care of the patient is in
caring for the patient." Francis W. Peabody [8, p. 882].

Not only is training to become a physician difficult; the standards of professional practice are also demanding. Physicians must be both scientifically and technically competent, as well as able to open up to the interests of patients. Physicians must be both astute and altruistic. Integrity, compassion, selfeffacement, courage, and self-sacrifice are all integral qualities to attaining the high professional standards expected in medical students and practicing physicians $[14,15]$. Unfortunately, stress, burnout, depression, or abuse of alcohol or other substances can sometimes complicate or even result from these high demands.

For a comprehensive clinical practice, it is not enough merely to learn and implement evidence-based biological, pharmacological, or surgical treatments. Connecting with, learning from, and caring for patients while taking into account their histories, attitudes, personal preferences, and wider range of needs are immensely rewarding parts of practice. This work is informed, in part, by knowledge and skills gleaned from the behavioral and social sciences. This broader humanistic approach to the whole patient may promote enhanced patient experiences and outcomes and also may serve to promote the quality and meaning of work for physicians, countering cynicism and professional burnout. There is anecdotal evidence that humanistic practice even serves as an antidote to professional burnout [16].

Ideally, we will build frameworks into our admission, curriculum, and assessment that select for and develop not only technical-scientific competencies but also humanistic ones.
Rather than just emphasizing quantitative skills, we should also require more qualitative analysis and related competencies. Narrative competence is a core example; we, as physicians, should have the ability to "recognize, absorb, interpret, and be moved by the stories one hears or reads" [17, p. 862]. In truly witnessing a patient's story, we convey that we can imagine his or her circumstance and validate his or her experience. This human interaction should be an entrustable professional activity. Narrative competence is central to the development of an authentic, empathic, reflective practitioner and directly correlates to humanism and professionalism [18, 19]. To engender this humanistic approach toward patient care, we must revise our current system-centered health care practice to allow for more time to both listen to and be with patients. This patient-centered approach builds upon the scientific basis of medicine and will require changes in the formal, informal, and hidden curricula. Faculty members must role model that getting to know patients and their story is important and something for which making time is valued. Additionally, assessment methods such as grading self-reflective writing and portfolios that map onto this competency must be validated. The MCAT2015 exam no longer includes the writing sample section that showed limited predictive validity on later performance [20]. One might wonder if in the future a writing section might return that assesses narrative competence. In addition to increasing the social, behavioral, and humanistic sciences into our curriculum and practice, and indeed into the MCAT2015, thoughtful planning is needed to allow for the additional time and reflective and metacognitive space required to foster truly patientcentered care.

\section{Recruitment into Psychiatry}

One possible positive effect of the enhancement of broader assessment methods in selecting students for medical school is that more people may be accepted with an interest in psychiatry. This effect would be welcomed, given the insufficient numbers of early-career psychiatry trainees, inadequate numbers of psychiatrists, and the prospect of a substantial reduction in the psychiatric workforce, given large numbers of older colleagues [21]. The valuing of behavioral and social sciences by the MCAT2015 may encourage more college students to pursue medicine who have these interests, and because psychiatry so values the humanities and social sciences, a greater pool of medical students may flow into psychiatry. Such interest, however, will only be sustained and fostered if the learning environment and the profession continue to value the basic, translational, and clinical neurosciences while also working to reduce stigma against mental illness and the specialty of psychiatry. 
Some empirical evidence supports the possibility that the MCAT2015 might enhance recruitment into psychiatry. Since 1987, Mount Sinai School of Medicine has recruited humanities and social science majors who have not completed standard premedical coursework and have not taken the MCAT [22]. In 2010 the Humanities and Medicine Program (HuMed) published a 6-year retrospective study [22] comparing this unique group of humanities and social sciences majors $(n=$ 85 ) and those who were more traditionally prepared and vetted for medical school $(n=606)$. Although this was a cohort study limited by the potential for confounding, HuMed students were twice as likely to receive honors in the psychiatry clerkship and more than twice as likely to pursue residencies in psychiatry and primary care than their non-HuMed counterparts.

Earlier emphasis on psychosocial behaviors in the medical education continuum provides us with the opportunity and challenge to be more influential as educators, mentors, and colleagues in the premedical years and beyond. Earlier and ongoing exposure to psychiatrists along this continuum may help increase the number of psychiatrists. Having psychiatrists teach and work with premedical and other undergraduates as early as the freshman year of college occurs only at some schools. In the preclerkship curriculum, psychiatrists have much to contribute in regard to the doctor-patient relationship and psychosocial factors that influence health and illness. At the clerkship and graduate medical education levels, psychiatrists have expanding roles to teach and work closely with primary care physicians and specialists and enhance teambased education and patient care. Taking on these roles may bring more people into the field of psychiatry and help correct the devaluation of psychiatry in medicine [23].

\section{Conclusions}

We welcome the addition of behavioral and social sciences content in the MCAT2015. This fundamental content places value on the pursuit of knowledge of psychological, social, and ethical concepts as integral to the practice of medicine as a biopsychosocial enterprise. Admissions processes should better align to recruit people with the educations, skills, and personal attributes suited to the biopsychosocial and humanistic nature of medicine. The MCAT2015 may be a step in this direction, while it also preserves and values the central role of basic and translational biomedical sciences. We suggest that these new assessments should be combined with innovative admissions instruments to assess not only cognitive skills in the behavioral and social sciences but also such core skills as narrative competency and self-reflective capacity explicitly. We hope the MCAT2015 will trigger a domino effect whereby we push further to align admission, complementary assessment methods, and medical school and residency curricula to include the correlates of humanism. Such a task will require planning, intention, and much time and money and will necessarily have major faculty development implications.

We have argued that learning the behavioral and social sciences and a broader humanistic approach to people, starting with collegiate studies as a requirement for entry into medical school and throughout medical training, should not only enhance patient experiences, and maybe outcomes, but also promote the quality and meaning of work for physicians. We hope it will make for a more socially and ethnically diverse workforce, rich in social and behavioral ideas. A biopsychosocial paradigm is relevant to preparing the whole physician workforce to better appreciate the psychosocial aspects of all diseases, to place the basic, translational, and clinical neurosciences in context, and to better understand and manage mental disorders. We also hope and anticipate that a greater number of medical students will be interested in becoming psychiatrists and that the image and status of psychiatry as a discipline will be improved. We will be well advantaged should any of these possible longer-term consequences of the MCAT2015 result in better care of our patients.

Acknowledgments The authors thank Gabrielle Termuehlen and Jennifer Pearlstein for their assistance in preparing this manuscript.

Disclosures Sylvia Bereknyei, DrPH, is a Research Scholar at the Stanford Center for Medical Education Research and Innovation and is a member of the MCAT PSBB Validity Committee. She is not acting as a representative of the Committee, and the views and opinions expressed here are her and her co-authors' own and not that of the AAMC or MCAT PSBB Validity Committee.

\section{References}

1. Schwartzstein RM, Rosenfield GC, Hilborn R, Oyewole SH, Mitchell K. Redesigning the MCAT exam; balancing multiple perspectives. Acad Med. 2013;88:560-7.

2. Association of American Medical Colleges. What's on the MCAT2015 Exam? Psychological, Social, and Biological Foundations of Behavior: Overview. Available at https://www.aamc. org/students/applying/mcat/map/mcat2015knowledgemapconcepts $2 /$ 369092/psbboverview.html. Accessed 15 Jan 2015.

3. Association of American Medical Colleges. MCAT2015 Frequently Asked Questions (FAQs): The Big Picture: How will the MCAT2015 exam better prepare doctors to work in a reformed health care system? Available at https://www.aamc.org/students/applying/mcat/ mcat2015/faqs/. Accessed 9 Jan 2015.

4. Kirch DG, Mitchell K, Ast C. The new 2015 MCAT. Testing competencies. JAMA. 2013;310:2243-4.

5. Kroopman M. AM last page: the MCAT exam: comparing the 1991 and 2015 exams. Acad Med. 2013;88:737.

6. Association of American Medical Colleges. MCAT2015 Frequently Asked Questions (FAQs): Cost and Length of the MCAT2015 Exam: Why is the MCAT2015 exam longer than the current MCAT exam? Available at https://www.aamc.org/students/applying/mcat/ mcat2015/faqs/. Accessed 15 Jan 2015. 
7. Association of American Medical Colleges. MCAT2015 Frequently Asked Questions (FAQs): Verbal Reasoning and the Writing Sample: How is the new exam's Critical Analysis and Reasoning Skills section different from the current Verbal Reasoning section? Available at https://www.aamc.org/students/applying/mcat/mcat2015/faqs/. Accessed 15 Jan 2015.

8. Peabody FW. The care of the patient. JAMA. 1927;88:877-82.

9. Engel CL. The need for a new medical model: a challenge for biomedical medicine. Science. 1977;196:129-36.

10. Association of American Medical Colleges. MedEdPORTAL iCollaborative. Pre-health Collection. Available at https://www. mededportal.org/icollaborative/about/initiatives/prehealth/. Accessed 12 Jan 2015.

11. Khan Academy. MCAT. Available at www.khanacademy.org/testprep/MCAT 2015. Accessed 12 Jan 2015.

12. Association of American Medical Colleges. Holistic Review. Advancing Holistic Review Initiative Purpose. Available at https:// www.aamc.org/initiatives/holisticreview/. Accessed 15 January 2015.

13. Association of American Medical Colleges. Holistic Review. What is holistic review? Available at https://www.aamc.org/initiatives/ holisticreview/. Accessed 15 January 2015.

14. McCullough LB. John Gregory and the invention of professional medical ethics and the profession of medicine. The Netherlands: Kluwer; 1988.
15. Coverdale J. Virtues-based advice for beginning medical students. Acad Psychiatry. 2007;31:354-7.

16. Chou CM, Kellom K, Shea JA. Attitudes and habits of highly humanistic physicians. Acad Med. 2014;89:1252-8.

17. Charon R. Narrative and medicine. N Engl J Med. 2004;350:862-4.

18. Charon R. The patient-physician relationship. Narrative medicine: a model for empathy, reflection, profession, and trust. JAMA. 2001;286:1897-901.

19. Wald HS, Davis SW, Reis SP, Monroe AD, Borkan JM. Reflecting on reflections: enhancement of medical education curriculum with structured field notes and guided feedback. Acad Med. 2009;84:830-7.

20. Gilbert GE, Basco Jr WT, Blue AV, O’Sullivan PS. Predictive validity of the medical college admissions test writing sample for the United States medical licensing examination steps 1 and 2. Adv Health Sci Educ Theory Pract. 2002;7(3):191-200.

21. Roberts LW, Ohayon M, Coverdale J, Goldsmith M, Beresin EV, Louie AK, et al. Strengthening psychiatry's numbers. Acad Psychiatry. 2013;37:293-6.

22. Muller D, Kase N. Challenging traditional premedical requirements as predictors of success in medical school: the Mount Sinai School of Medicine Humanities and Medicine Program. Acad Med. 2010;85: $1378-83$.

23. Roberts LW, Maldonado Y, Coverdale J, Balon R, Louie AK, Beresin EV. The critical need to diversify the clinical and academic workforce. Acad Psychiatry. 2014;38:394-7. 